ORIGINAL

\title{
Effect of different levels of L-carnitine and lysine-methionine on broiler blood parameters
}

\author{
Efecto de suplementación con L-carnitina y lisina-metionina sobre \\ parámetros de sangre de pollos de engorde
}

\author{
Babak Hosseintabar, ${ }^{1}$ M.Sc, Mohammad Dadashbeiki, ${ }^{2}$ DVM, Mehrdad Bouyeh, ${ }^{1}$ Ph.D, \\ Alireza Seidavi, ${ }^{1 *}$ Ph.D, René van den Hoven, ${ }^{3}$ Ph.D, Sandra Gamboa, ${ }^{4,5}$, Ph.D.
}

\begin{abstract}
${ }^{1}$ Islamic Azad University, Rasht Branch, Department of Animal Science, Rasht, Iran, ${ }^{2}$ Islamic Azad University, Rasht Branch, Department of Veterinary Science, Rasht, Iran, ${ }^{3}$ Vetmeduni Wien, Vienna, Austria, ${ }^{4}$ Polytechnic Institute, Agricultural School, Department of Zootechnic Sciences, Animal Reproduction Laboratory, Coimbra, Portugal, ${ }^{5}$ CERNAS- Centre for Studies on Natural Resources, Environment and Society, Polytechnic Institute, Coimbra, Portugal. *Correspondence: alirezaseidavi@iaurasht.ac.ir
\end{abstract}

Received: June 2014; Accepted: December 2014.

\begin{abstract}
Objetive. In the present study a completely randomized $3 \times 3$ factorial design was used to analyze the effects of different levels of L-Carnitine, lysine(Lys) and methionine (Met) on the blood concentrations of energy, protein and lipid metabolites of male broiler chickens. Materials and methods. A total of 270 newly hatched male broiler chickens (Ross 308) were randomly assigned to 9 groups (ten broilers per replicate and three replicates per treatment). The control group was fed a basal diet, whereas the treatment groups were fed basal diets supplemented with L-Carnitine $(0 \mathrm{mg} / \mathrm{kg}, 75 \mathrm{mg} / \mathrm{kg}$ and $150 \mathrm{mg} / \mathrm{kg}$ ) and lysine-methionine (0,15 and 30\%) for 42 days. On day 42, one bird was randomly chosen per replication, a blood sample was taken and the blood concentrations of glucose (GLU), uric acid (UAc), triglyceride (TG), VLDL, HDL, LDL, total protein (TP), albumin (Alb) and total cholesterol (TC) were analyzed. Results. Dietary L-carnitine supplementation had a significant effect $(p<0.05)$ on uric acid (UAC), HDL, LDL, and total cholesterol (TC). The birds feed L-carnitine plus Lys and Met presented the highest plasmatic UAc level and the lowest plasmatic TC and LDL level. Moreover, L-carnitine significantly reduced total cholesterol (TC) when compared with both the control group and the birds feed Lys and Met without L-carnitine. Conclusions. A diet with $150 \mathrm{mg} / \mathrm{kg} \mathrm{L-carnitine}$ plus $15 \%$ Lys and Met seems to be enough to sustain low plasmatic TC, LDL and HDL concentrations on male broiler.
\end{abstract}

Key words: amino acids, broiler chicken,lipid profile, serum metabolites, supplementation (Source: $C A B$ ).

\section{RESUMEN}

Objetivo. Se realizó un estudio para determinar el efecto de la suplementación deL-carnitina, lisina (Lys) y metionina (Met) sobre los metabolitos sanguíneos de pollos de engorde. Materiales y métodos. Se utilizaran 270 pollos de la línea Ross 308 de un día de edad y se dividieron en 9 tratamientos en un diseño al azar con arreglo factorial 3x3: tres niveles de L-carnitina $(0 \mathrm{mg} / \mathrm{kg}, 75 \mathrm{mg} / \mathrm{kg}$ y 150 $\mathrm{mg} / \mathrm{kg}$ ) y tres de lisina-metionina $(0,15$ y $30 \%)$ durante 42 días. Cada tratamiento constó de 3 repeticiones con 10 pollos por repetición. El día 42 de edad, se tomaron muestras de sangre de tres aves por tratamiento para cuantificar niveles séricos de glucosa (GLU), ácido úrico (UAC), triglicéridos (TG), VLDL, HDL, LDL, proteínas totales (PT), albúmina (Alb) y colesterol total (TC). Resultados. La suplementación de L-carnitina en la dieta tuvo un efecto significativo $(p<0.05)$ en los niveles de ácido úrico en suero (UAC), HDL, LDL y colesterol total (CT). Las aves alimentadas con L-carnitina más Lys y 
Met mostraron niveles séricos más altos de UAc y menor TCy LDL. Por otra parte, la L-carnitina redujó significativamente el colesterol total (CT), cuando se comparó con el grupo control y con los pollos alimentados con Lys y Met, sin L-carnitina. Conclusiones. Una dieta con $150 \mathrm{mg} / \mathrm{kg}$ de L-carnitina y $15 \%$ Lys y Met parece ser suficiente para mantener bajas concentraciones plasmáticas de TC, LDL y HDL en pollos de engorde.

Palabras clave: Aminoácidos, metabolitos séricos, perfil lipídico, pollos, suplementación (Fuente: CAB).

\section{INTRODUCTION}

The increase in world population and the increase in household purchasing power are the factors that determine the future consumption of meat. Per capita, the global meat consumption is around $14 \mathrm{~kg}$ per year and the trend is upwards. The poultry belongs to the types of meat which can be produced relatively efficiently with low environmental impact (1). Moreover, the poultry meat has maintained a higher value compared to other species for several reasons including nutritional aspects as low-fat with high unsaturation degree of fatty acids and low cholesterol level.

The growing demand for poultry meat has resulted in pressure on breeders to increase the growth rate of birds, feed efficiency, size of breast muscle and reduction in abdominal fatness (2). Therefore, research is being directed to further improve techniques of poultry meat production. The improvement in carcass composition with additives has become a focus on nutrition research. The addition of amino acids and metabolic intermediates to diets may lower the abdominal fat deposition in poultry. L-carnitine, the biological active form of carnitine, is a quaternary ammonium compound synthesized in the liver, kidney and brain (3), from the essential amino acids lysine (Lys) and methionine (Met).

The effects of dietary L-carnitine, Lys and Met supplementations on the growth performance and body composition of broiler chickens are still poorly understood. Some authors reported that abdominal fat deposition in broilers is reduced by L-carnitine supplementation without significant effect on daily gain or feed conversion (4), while others found no impact of dietary L-carnitine supplementation on abdominal fat composition (5). However, Bouyeh and Gevorgyan (6) and Celik et al (7) showed that growth performance of broilers was improved by L-carnitine supplementation. Regarding Lys and Met supplementation in excess of NRC recommendations, Mukhtar et al (8) found a significant improvement in feed intake, average body weight gain and feed conversion ratio, while $\mathrm{Si}$ et al (9) concluded that it should not be elevate the level of Met if Lys is in excess of its minimum needs.

\section{INTRODUCCIÓN}

El incremento de la población mundial y el incremento del poder de compra de los hogares son factores que determinan el consumo futuro de carne. Per cápital, el consumo global de carne es de aproximadamente $14 \mathrm{~kg}$ por año y la tendencia es hacia arriba. Las aves son el tipo de carne que puede ser producido de manera relativamente eficiente con un bajo impacto ambiental (1). Más aún, la carne de aves ha mantenido un alto valor comparado con otras especies por varias razones incluyendo aspectos nutricionales como baja grasa con alto grado de saturación de ácidos grasos y bajo nivel de colesterol.

La demanda creciente por carne de aves ha resultado en presión sobre los criadores para incrementar la tasa de crecimiento de las aves, la eficiencia alimentación, la talla del músculo del pecho y la reducción en la grasa abdominal (2). Por esto, la investigación se dirige hacia cómo mejorar las técnicas de producción de carne de aves. La mejora en la composición de la carcasa con aditivos ha sido el foco de investigaciones nutricionales. La adición de aminoácidos e intermediarios metabólicos a las dietas puede reducir la deposición abdominal de grasa en aves. L-carnitine, la forma activa biológica de carnitine, es un compuesto cuaternario de amonio sintetizado en el hígado, riñones y cerebro (3), de aminoácidos esenciales lisina (Lys) y metionina (Met).

Los efectos de suplementos dietarios de L-carnitine, Lys y Met sobre el desempeño de crecimiento y composición del cuerpo de pollos De engorde son aún poco comprendidos. Algunos autores reportaron que la deposición de grasa abdominal en los pollos de engorde se reduce por suplementos de L-carnitine sin ningún efecto significativo sobre la ganancia diaria de la conversión de alimentación (4), mientras otros no encontraron ningún impacto dietario del suplemento de L-carnitine sobre la composición de grasa abdominal (5). Sin embargo, Bouyeh y Gevorgyan (6) y Celik et al (7) demostraron que el desempeño de crecimiento en pollos de engorde mejoró con el suplemento de L-carnitine. Con relación al suplemento de Lys y Met en exceso de las recomendaciones de la NRC, Mukhtar et al (8) encontró una mejora significativa en la ingestión de alimentos, la ganancia promedio de peso corporal y la tasa de conversión de 
Corduk et al (10) have demonstrated that addition of L-carnitine to diet did not significantly affect body weight gain, feed intake, feed conversion ratio and blood parameters in broiler chickens. However, Parizadian et al (11) found that supplementing diet with L-carnitine will reduce blood triglyceride, cholesterol and improve egg quality in laying Japanese quail. Therefore, lower body fat deposition may be attributed to increased fat catabolism or diminished endogenous fatty acid synthesis or both processes (12).

The bioavailability of L-carnitine varies due to dietary composition but since it can be biosynthesized endogenously from methionine and lysine, these two amino acids are usually the more important limiting amino acids in poultry nutrition. Methionine and lysine are frequently supplemented in the formulated diets. Previous studies showed that methionine supplement during the early stages of chicken growth causes significant improvement in hematocrit, red blood cell count and related parameters $(13,14)$.

Theoretically, supplementing the broiler diet with an adequate content of L-carnitine would facilitate the $\beta$-oxidation of fatty acids, and decrease esterification reactions and triacylglycerol storage in the adipose tissue (12). As blood biochemistry may monitor the quality of nutrition and health of birds $(13,15)$, we have quantified serum markers of lipid and protein metabolism on 42 day older male broiler chickens feed extra levels of dietary Lys-Met with and without L-carnitine.

The objetive of this study was to evaluate the effects of different levels of L-carnitine, Lys and Met on the blood concentrations of energy proteins and lipid metabolites of male broiler chickens.

\section{MATERIALS AND METHODS}

Study site. This study was developed in the poultry farm facilities of the Agriculture Faculty of Islamic Azad University, in Rasht, Iran. It is situated $7 \mathrm{~m}$ below sea level and located at latitude $37^{\circ} 15^{\prime} \mathrm{N}$ and longitude $49^{\circ} 36^{\prime} \mathrm{E}$.

Facilities. The facilities $(20 \times 40$ meters $)$ included, other than the main compartment for chicken, a storage room, where the ingredients are grinded and mixed. Six ventilators were used to maintain an air discharge power of 1400 cubic meters per hour. During the experiments, room temperature was maintained applied according to the instructions of the Ross 208 strain rearing manual. The temperature was adjusted to approximately $33^{\circ} \mathrm{C}$ on the day before day 1 . The birds were housed in the main compartment, in alimentación, mientras que Si et al (9) concluyó que no se debería elevar el nivel de Met si Lys está en exceso de sus necesidades mínimas.

Corduk et al (10) han demostrado que la adición de L-carnitine a la dieta no afectó de manera significativa la ganancia de peso corporal, ingesta de alimento, tasa de conversión de alimento y parámetros de sangre en pollos de engorde. Sin embargo, Parizadian et al (11) encontraron que suplementar la dieta con L-carnitine reduce los triglicéridos en la sangre, el colesterol y mejora la calidad de postura de huevos en codornices japonesas. Por lo tanto, una baja deposición de grasa corporal puede atribuirse a un incremento en el catabolismo de grasa o síntesis de ácidos grasos en disminuida o ambos procesos (12).

La biodisponibilidad de L-carnitine maría debido a la composición dietario pero cómo puede ser biosintetizada de manera endógena de metionina y lisina, estos dos aminoácidos son usualmente los aminoácidos en limitantes más importantes en la nutrición de pollos. La metionina y la lisina son frecuentemente suplementados en dietas formuladas. Estudios previos muestra que el suplemento de metionina durante Las primeras fases de crecimiento de pollos causa mejora significativa en hematocritos, conteo de glóbulos rojos y parámetros relacionados $(13,14)$

Teóricamente, suplementar la dieta del pollo de engorde con un contenido adecuado de L-carnitine podría facilitar la $\beta$-oxidación de ácidos grasos, y una disminución de reacciones de esterificación $Y$ almacenamiento de traicilglicerol en el tejido adiposo (12). Ya que la bioquímica de la sangre puede monitorear la calidad de la nutrición y la salud de las aves $(13,15)$, hemos cuantificado los marcadores de suero del metabolismo de lípidos y proteínas en pollos de engorde machos de alimentación de pollos de 42 días con niveles dietarios extra de Lys-Met sin L-carnitine.

El objetivo de este estudio fue evaluar los efectos de distintos niveles de L-carnitine, Lys y Met en las concentraciones en sangre de proteínas de energía y metabolitos lípidos en pollos de engorde machos.

\section{MATERIALES Y MÉTODOS}

Locación de estudio. Este estudio se desarrolló en las instalaciones de cría de pollos de la facultad de Agricultura de la universidad islámica de Azad, en Rasht, Irán. Está ubicada $7 \mathrm{~m}$ bajo el nivel del mar y localizada a una latitud de $37^{\circ} 15^{\prime}$ norte y longitud de 49³6' este.

Instalaciones. Las instalaciones $(20 \times 40 \mathrm{~m})$ incluye, además del compartimiento principal 
27 pens with $1.0 \times 2 \mathrm{~m}$. Prior to the experiment the hall and all equipment was carefully cleaned and disinfected according to general bio-security procedures for broiler production. Temperature, light, ventilation, drinkers and feeders were all managed according to the Ross 208 manual for production and were equal for all treatments.

Animals and experimental design. A total of 270 newly hatched male Ross 308 broilers were used for the experiment. The birds had been sourced from the Ross hatchery in Iran. After sexing, animals were randomly allocated to the experimental units. A total of 9 treatments groups were formed (Table 1). Each group had 3 replicates with 10 birds per replicate. Animals were reared for 42 days:birds received starter diet from 1-21 d, grower diet from 22-35 d and finisher diet from 36-42 d of age, respectively. The L-carnitine, Lys and Met levels were according to NRC (16) recommendations for starter (1.1Lys and $0.9 \mathrm{Met}$ ), grower (1.0 Lys and $0.72 \mathrm{Met}$ ) and finisher (0.8 Lys and 0.9 Met) feed.

The control group ration was the basis of all other diets (Table 2). In the experimental diets, extra L-carnitine, Lys and Met were added in excess of NRC (16) recommendations according to table 2.

Sampling and blood parameters. At the end of the experiment, at42 days-of-age, one bird per group was randomly selected for blood collection. Blood samples ( $1 \mathrm{ml} /$ bird) were collected using a $2 \mathrm{ml}$ syringe and left to stand at $30^{\circ} \mathrm{C}$ to allow clotting and clot retraction. These rum that remained after clotting was centrifuged at $3000 \mathrm{rpm}$ for 10 minutes at room temperature. Blood parameters analyzed in serum were glucose (GLU), uric acid (UAc), total triglycerides (TG), total cholesterol (TC), total protein (TP), albumin (Alb), high density lipoprotein (HDL), low density lipoprotein (LDL)and very low density lipoprotein (VLDL). The concentrations for these parameters were determined by routine methods using commercial laboratory kits (Pars Azmoon Co., Tehran, Iran), according to the manufacturer's instructions. GLU was measured with an enzymatic, colorimetric glucose oxidaseperoxidase (GOD-POD) method, while TC, TG, HDL, LDL and VLDL were determined by enzymatic CHOD-PAP assays. Alb was determined based on the bromocresol green method, whilst the UAc was determined by enzymatic methods para pollos, un cuarto de almacenamiento, donde los ingredientes fueron molidos y mezclados. Seis ventiladores fueron utilizados para mantener un poder de descarga de aire de $1400 \mathrm{~m}^{3}$ por hora. Durante los experimentos, la temperatura Del cuarto se mantuvo aplicada de acuerdo las instrucciones del manual de cría de cepa Ross 208. La temperatura sea justo aproximadamente $33^{\circ} \mathrm{C}$ el día antes del día uno. La sabes fueron alojadas en el compartimiento principal, en 27 corrales de $1 \mathrm{~m}$ por $2 \mathrm{~m}$. Antes de iniciar el experimento el corredor y todo el equipo fue cuidadosamente limpiado y desinfectado de acuerdo a los procedimientos generales de bioseguridad para producción de pollos de engorde. La temperatura, luz, ventilación, bebederos y comederos fueron todos manejados de acuerdo al manual para producción Ross 208 Y fueron iguales para todos los tratamientos.

Animales y diseño de experimento. Un total de 270 pollos de engorde machos Ross 308 recién nacidos fueron utilizados para el experimento. Los pollos proceden del criadero Ross en Irán. Luego de determinar el sexo, los animales fueron asignados de manera aleatoria a las unidades experimentales. Un total de 9 grupos de tratamiento fueron formados (Tabla 1). Cada grupo tiene 3 replicados con 10 aves por replicado. Los animales fueron criados por 42 días; las aves recibieron una dieta de inicio desde el día 1 al 21, dieta de crecimiento del día 22 al 35 y dieta de terminación del día 36 al 42 , respectivamente. Los niveles de L-carnitine, Lys y Met estuvieron de acuerdo a las recomendaciones de alimentación de NRC (16) para inicio (1.1 Lys y 0.9 Met), crecimiento (1.0 Lys y 0.72 Met) y terminación (0.8 Lys y 0.9 Met).

La ración del grupo de control fue la base de las otras dietas (Tabla 2). En las dietas experimentales, extra L-carnitine, Lys y Met fueron adicionados en exceso de las recomendaciones NRC (16) de acuerdo a la tabla 2.

Muestreo y parámetros de sangre. Al final del experimento, a los 42 días de edad, un ave por grupo fue seleccionada de manera aleatoria para recolección de sangre. Muestras de sangre $(1 \mathrm{ml}$ por ave) fueron recolectadas utilizando una jeringa de $2 \mathrm{ml} \mathrm{Y}$ se dejó reposar a $30^{\circ} \mathrm{C}$ para permitir coagulación y retracción de cuadros. El resultado después de la cual culo acción fue centrifugado a 3000 revoluciones por minuto por 10 minutos a temperatura ambiente. Los parámetros de sangre

Table 1. The amount of L-carnitine, lysine (Lys) and methionine (Met) in each treatment.

\begin{tabular}{lccccccccc}
\hline & \multicolumn{10}{c}{ Treatments } \\
\cline { 2 - 7 } & T1 & T2 & T3 & T4 & T5 & T6 & T7 & T8 \\
\hline L-carnitine & NRC & NRC & NRC & $75 \mathrm{mg} / \mathrm{kg}$ & $75 \mathrm{mg} / \mathrm{kg}$ & $75 \mathrm{mg} / \mathrm{kg}$ & $150 \mathrm{mg} / \mathrm{kg}$ & $150 \mathrm{mg} / \mathrm{kg}$ & $150 \mathrm{mg} / \mathrm{kg}$ \\
Lysine & $\mathrm{NRC}$ & $\mathrm{NRC}+15 \%$ & $\mathrm{NRC}+30 \%$ & $\mathrm{NRC}$ & $\mathrm{NRC}+15 \%$ & $\mathrm{NRC}+30 \%$ & $\mathrm{NRC}$ & $\mathrm{NRC}+15 \%$ & $\mathrm{NRC}+30 \%$ \\
Methionine & $\mathrm{NRC}$ & $\mathrm{NRC}+15 \%$ & $\mathrm{NRC}+30 \%$ & $\mathrm{NRC}$ & $\mathrm{NRC}+15 \%$ & $\mathrm{NRC}+30 \%$ & $\mathrm{NRC}$ & $\mathrm{NRC}+15 \%$ & $\mathrm{NRC}+30 \%$ \\
\hline
\end{tabular}


Table 2. The ingredients (\%) and nutrient constitution of the basal diets used in this study for the starter $\left(1^{\text {st }}-21^{\text {st }}\right.$ days of age $)$, grower $\left(22^{\text {nd }}-\right.$ $35^{\text {th }}$ days of age $)$, and finisher $\left(36^{\text {th }}-42^{\text {nd }}\right.$ days of age) periods.

\begin{tabular}{|c|c|c|c|}
\hline Ingredient (\%) & $\begin{array}{l}\text { Starter } \\
\text { period }\end{array}$ & $\begin{array}{c}\text { Finisher } \\
\text { period }\end{array}$ & $\begin{array}{c}\text { Finisher } \\
\text { period }\end{array}$ \\
\hline Corn & 59.0 & 62.0 & 65.0 \\
\hline Soybean Meal & 35.0 & 30.0 & 27.0 \\
\hline Soybean oil & 2.0 & 3.5 & 3.5 \\
\hline $\mathrm{Ca} \% 22 \mathrm{P} \% 18$ & 1.5 & 1.8 & 1.7 \\
\hline Mineral oysters & 1.2 & 1.5 & 1.5 \\
\hline $\mathrm{NaCl}$ & 0.3 & 0.2 & 0.2 \\
\hline Lysine-Hydro-Chloride & 0.1 & 0.1 & 0.15 \\
\hline DL-Methionine & 0.2 & 0.2 & 0.25 \\
\hline Mineral premix* & 0.3 & 0.3 & 0.3 \\
\hline Vitamin premix** & 0.3 & 0.3 & 0.3 \\
\hline $\begin{array}{l}\text { Sodium bicarbonate } \\
\text { (NaHCO3) }\end{array}$ & 0.1 & 0.1 & 0.1 \\
\hline $\begin{array}{l}\text { Metabolizable energy } \\
(\mathrm{kcal} / \mathrm{kg})\end{array}$ & 2897.8 & 2955 & 3058 \\
\hline Crude protein (\%) & 21.7 & 20.8 & 18.25 \\
\hline Crude fiber (\%) & 2.26 & 2.22 & 2.14 \\
\hline Calcium (\%) & 0.80 & 0.80 & 0.79 \\
\hline Available Potassium (\%) & 0.48 & 0.46 & 0.42 \\
\hline Sodium (\%) & 0.15 & 0.15 & 0.15 \\
\hline Lysine (\%) & 1.41 & 1.26 & 1.22 \\
\hline Methionine (\%) & 0.61 & 0.57 & 0.48 \\
\hline Energy: Protein ratio & 133.58 & 142 & 167 \\
\hline Ca: P & 1.66 & 1.75 & 1.88 \\
\hline Linoleic Acid (\%) & 2.46 & 2.73 & 2.95 \\
\hline Methionine+Cysteine (\%) & 0.97 & 0.91 & 0.77 \\
\hline
\end{tabular}

*Calcium Pantothenate: $4 \mathrm{mg} / \mathrm{g}$; Niacin: $15 \mathrm{mg} / \mathrm{g}$; Vitamin B6: $13 \mathrm{mg} / \mathrm{g}$; Cu: $3 \mathrm{mg} / \mathrm{g}$; Zn: $15 \mathrm{mg} / \mathrm{g}$; Mn: $20 \mathrm{mg} / \mathrm{g}$; Fe: $10 \mathrm{mg} / \mathrm{g} ; \mathrm{K}: 0.3 \mathrm{mg} / \mathrm{g}$ **Vitamin A: $5000 \mathrm{IU} / \mathrm{g}$; Vitamin D3: $500 \mathrm{IU} / \mathrm{g}$; Vitamin E: $3 \mathrm{mg} / \mathrm{g}$; Vitamin $\mathrm{K} 3: 1.5 \mathrm{mg} / \mathrm{g}$; Vitamin B2: $1 \mathrm{mg} / \mathrm{g}$

using the uricase-TOOS method and TP was assayed by the Biuret method.

Statistical analysis. For the $3 \times 3$ factorial design the linear model $Y_{i j k}=\mu+a_{j}+\beta_{k}+(a \beta)_{j k}+\varepsilon_{i j k}$ was applied, in which the value of each observation $(Y)$ relies on the population effects average $(\mu)$ plus the effect of factor $A\left(a_{j}, L\right.$-carnitine level), the effect of factor $B\left(\beta_{k}\right.$, methionine-lysine level) and their interaction $\left[(a \beta)_{j k}\right]$, and the experimental error $(\varepsilon)$. A general mixed model was chosen as most robust statistical test that respected the repeated measures. The replicate was the experimental unit (17).

\section{RESULTS}

The results for the biochemical parameters concentration at day 42 in broilers plasma according to feed treatments are summarized in table 3 . analizados en el suero fueron glucosa (GLU), ácido úrico (UAc), triglicéridos totales (TG), colesterol total (TC), proteína total (TP), albúmina (Alb), lipoproteína de alta densidad (HDL), lipoproteína de baja densidad (LDL) y lipoproteína de muy baja densidad (VLDL). Las concentraciones para estos parámetros se determinaron por métodos rutinarios utilizando kits comerciales de laboratorio (Pars Amonn Co., Teherán, Irán), de acuerdo a las instrucciones del fabricante. El GLU fue medido con el método enzimático, colorimétrico de glucosa oxidasa y peroxidasa (GOD-POD), mientras que el TC, TG, HDL, LDL y VLDL fueron determinados por análisis enzimático CHOD-PAP. El Alb fue determinado con base en el método de bromocresol verde, mientras que el UAc se determinó con métodos enzimáticos utilizando el método uricasaTOOS y el TP se analizó con el método Biuret.

Análisis Estadístico. Para el diseño factorial $3 \times 3$ se aplicó el modelo $Y_{i j k}=\mu+a_{j}+\beta_{k}+(a \beta)_{j k}+\varepsilon_{i j k}$, en el que el valor de cada observación $(Y)$ depende en el promedio de efectos en la población $(\mu)$ más el efecto del factor A (nivel de $a_{j}$, $L$-carnitine), el efecto del facto $B\left(\beta_{k}\right.$ nivel de metionina lisina) y su interacción $\left[(a \beta)_{j k}\right]$, y el error experimental $(\varepsilon)$. Un modelo mixto general se escogió como la prueba estadística más robusta que respetaba las medidas repetidas. El replicado fue la unidad experimental (17).

\section{RESULTADOS}

Los resultados para la concentración de parámetros biomédicos en el día 42 en el plasma de pollos de engorde de acuerdo a los tratamientos de alimentación se resumen en la tabla 3.

En el día 42, no hubo cambios significativos en las concentraciones medias de parámetros de sangre entre aves sometidas a suplementación extra LysMet sin extra L-carnitine (T2 y T3) y el grupo de control (T1). Sin embargo, la suplementación con Lys-Met sola mostró una disminución discreta en el colesterol total (TC) plasmático y la lipoproteína de baja densidad (LDL) cuando se comparó con la dieta de control (T1)(Tabla 3). No hubo efectos principales evidentes para suplementación de Lys más Met a través de los tratamientos (T4).

La suplementación de la dieta con L-carnitine y Lys más Met no tuvo efecto ( $p>0.05)$ en glucosa plasmática (GLU), triglicéridos (TG), proteína total (TP), Albúmina (Alb) y lipoproteína de baja densidad (LDL). Por el contrario, el efecto en tratamientos sobre el ácido úrico plasmático (UAc), colesterol (TC), lipoproteína de baja densidad (LDL) y lipoproteína de alta densidad (HDL) mue significativo $(p<0.05)$ (Tabla 3). Efectos principales fueron evidentes para suplementación de L-carnitine 
Table 3. Mean values ( \pm s.e.m.) on blood parameters of broilers diets differing in dietary L-carnitine, lysine (Lys) and methionine (Met) levels.

\begin{tabular}{|c|c|c|c|c|c|c|c|c|c|c|c|}
\hline \multirow{2}{*}{$\begin{array}{c}\text { Blood } \\
\text { parameters }\end{array}$} & \multicolumn{9}{|c|}{ Treatments $^{1}$} & \multirow{2}{*}{ s.e.m. } & \multirow{2}{*}{ Sig } \\
\hline & T1 & T2 & T3 & T4 & T5 & T6 & T7 & T8 & T9 & & \\
\hline $\begin{array}{l}\text { Glucose } \\
{[\mathrm{mg} / \mathrm{dl}]}\end{array}$ & $\begin{array}{c}235^{\mathrm{a}} \\
\pm 4.35\end{array}$ & $\begin{array}{c}233.67^{\mathrm{a}} \\
\pm 8.32\end{array}$ & $\begin{array}{c}239.33^{a} \\
\pm 8.38\end{array}$ & $\begin{array}{l}248.67^{a} \\
\pm 28.20\end{array}$ & $\begin{array}{c}249^{a} \\
\pm 9.84\end{array}$ & $\begin{array}{c}240.67^{a} \\
\pm 4.72\end{array}$ & $\begin{array}{l}253.30^{\mathrm{a}} \\
\pm 21.5\end{array}$ & $\begin{array}{c}254^{\mathrm{a}} \\
\pm 24.24\end{array}$ & $\begin{array}{c}234^{\mathrm{a}} \\
\pm 9.53\end{array}$ & 9.06 & ns \\
\hline $\begin{array}{l}\text { Uric acid } \\
{[\mathrm{mg} / \mathrm{dl}]}\end{array}$ & $\begin{array}{l}2.23^{a} \\
\pm 1.60\end{array}$ & $\begin{array}{l}2.63 \mathrm{ab} \\
\pm 0.45\end{array}$ & $\begin{array}{l}2.63^{\mathrm{ab}} \\
\pm 1.17\end{array}$ & $\begin{array}{l}3.26^{\mathrm{ab}} \\
\pm 1.90\end{array}$ & $\begin{array}{l}4.20^{\mathrm{ab}} \\
\pm 0.52\end{array}$ & $\begin{array}{l}4.70^{\mathrm{ab}} \\
\pm 1.21\end{array}$ & $\begin{array}{l}4.70^{\mathrm{ab}} \\
\pm 1.86\end{array}$ & $\begin{array}{l}4.63^{\mathrm{ab}} \\
\pm 1.90\end{array}$ & $\begin{array}{l}4.96^{b} \\
\pm 0.75\end{array}$ & 0.78 & $*$ \\
\hline $\begin{array}{l}\text { Triglyceride [mg/ } \\
\text { dl] }\end{array}$ & $\begin{array}{c}132^{\mathrm{a}} \\
\pm 13.50\end{array}$ & $\begin{array}{l}149.67^{a} \\
\pm 20.50\end{array}$ & $\begin{array}{l}106.67^{a} \\
\pm 22.36\end{array}$ & $\begin{array}{l}139.00^{\mathrm{a}} \\
\pm 25.51\end{array}$ & $\begin{array}{l}142.00^{\mathrm{a}} \\
\pm 17.05\end{array}$ & $\begin{array}{l}115.30^{\mathrm{a}} \\
\pm 40.5\end{array}$ & $\begin{array}{c}135.3^{\mathrm{a}} \\
\pm 12\end{array}$ & $\begin{array}{l}121.67^{a} \\
\pm 23.50\end{array}$ & $\begin{array}{l}137.33^{a} \\
\pm 17.15\end{array}$ & 13.17 & ns \\
\hline $\begin{array}{l}\text { Protein } \\
{[\mathrm{mg} / \mathrm{dl}]}\end{array}$ & $\begin{array}{l}3.03^{a} \\
\pm 0.37\end{array}$ & $\begin{array}{l}3.16^{\mathrm{a}} \\
\pm 0.32\end{array}$ & $\begin{array}{l}3.26^{a} \\
\pm 0.05\end{array}$ & $\begin{array}{l}3.33^{\mathrm{a}} \\
\pm 0.58\end{array}$ & $\begin{array}{l}3.26^{\mathrm{a}} \\
\pm 0.25\end{array}$ & $\begin{array}{l}3.53^{a} \\
\pm 0.65\end{array}$ & $\begin{array}{l}3.46^{a} \\
\pm 0.60\end{array}$ & $\begin{array}{l}3.26^{\mathrm{a}} \\
\pm 0.51\end{array}$ & $\begin{array}{l}3.53^{a} \\
\pm 0.46\end{array}$ & 0.26 & ns \\
\hline $\begin{array}{l}\text { Albumin } \\
{[\mathrm{mg} / \mathrm{dl}]}\end{array}$ & $\begin{array}{l}1.36^{\mathrm{a}} \\
\pm 0.25\end{array}$ & $\begin{array}{l}1.36^{a} \\
\pm 0.25\end{array}$ & $\begin{array}{c}1.6^{\mathrm{a}} \\
\pm 0.10\end{array}$ & $\begin{array}{l}1.36^{\mathrm{a}} \\
\pm 0.15\end{array}$ & $\begin{array}{l}1.53^{a} \\
\pm 0.11\end{array}$ & $\begin{array}{l}1.56^{\mathrm{a}} \\
\pm 0.32\end{array}$ & $\begin{array}{r}1.56^{\mathrm{a}} \\
\pm 0.15\end{array}$ & $\begin{array}{l}1.43^{\mathrm{a}} \\
\pm 0.28\end{array}$ & $\begin{array}{l}1.66^{\mathrm{a}} \\
\pm 0.23\end{array}$ & 0.12 & ns \\
\hline $\begin{array}{l}\text { Cholesterol [mg/ } \\
\mathrm{dl}]\end{array}$ & $\begin{array}{l}293.30^{a} \\
\pm 6.10\end{array}$ & $\begin{array}{c}284^{\mathrm{a}} \\
\pm 61.53\end{array}$ & $\begin{array}{c}271^{\mathrm{a}} \\
\pm 9.53\end{array}$ & $\begin{array}{c}185^{\mathrm{b}} \\
\pm 49.86\end{array}$ & $\begin{array}{l}133^{\mathrm{bc}} \\
\pm 10\end{array}$ & $\begin{array}{c}142^{\mathrm{bc}} \\
\pm 12.70\end{array}$ & $\begin{array}{c}135.67^{\mathrm{bc}} \\
\pm 4.90\end{array}$ & $\begin{array}{l}115.3^{c} \\
\pm 9.80\end{array}$ & $\begin{array}{c}131.30^{c} \\
\pm 20.8\end{array}$ & 16.35 & $*$ \\
\hline $\begin{array}{l}\mathrm{LDL} \\
{[\mathrm{mg} / \mathrm{dl}]}\end{array}$ & $\begin{array}{c}169.30^{\mathrm{a}} \\
\pm 8.90\end{array}$ & $\begin{array}{c}165^{\mathrm{a}} \\
\pm 49.36\end{array}$ & $\begin{array}{l}158.33^{a} \\
\pm 14.64\end{array}$ & $\begin{array}{l}71.30^{\mathrm{b}} \\
\pm 62.04\end{array}$ & $\begin{array}{c}81^{\mathrm{b}} \\
\pm 6.24\end{array}$ & $\begin{array}{l}24.60^{c} \\
\pm 5.85\end{array}$ & $\begin{array}{l}21.00^{c} \\
\pm 5.29\end{array}$ & $\begin{array}{l}12.67^{c} \\
\pm 3.50\end{array}$ & $\begin{array}{l}15.67^{c} \\
\pm 15.14\end{array}$ & 16.01 & $* *$ \\
\hline $\begin{array}{l}\mathrm{HDL} \\
{[\mathrm{mg} / \mathrm{dl}]}\end{array}$ & $\begin{array}{c}88^{\mathrm{a}} \\
\pm 8.23\end{array}$ & $\begin{array}{c}89^{\mathrm{ab}} \\
\pm 16.09\end{array}$ & $\begin{array}{c}91.60^{\mathrm{ab}} \\
\pm 7.09\end{array}$ & $\begin{array}{l}85.60^{\mathrm{ab}} \\
\pm 10.01\end{array}$ & $\begin{array}{c}86.60^{\mathrm{ab}} \\
\pm 1.10\end{array}$ & $\begin{array}{l}94.30^{\mathrm{ab}} \\
\pm 10.20\end{array}$ & $\begin{array}{c}87.33^{\mathrm{ab}} \\
\pm 6.11\end{array}$ & $\begin{array}{l}78.30^{\mathrm{b}} \\
\pm 10.4\end{array}$ & $\begin{array}{l}97.3^{\mathrm{ab}} \\
\pm 10.14\end{array}$ & 5.44 & $*$ \\
\hline $\begin{array}{l}\text { VLDL } \\
{[\mathrm{mg} / \mathrm{dl}]}\end{array}$ & $\begin{array}{l}26.66^{a} \\
\pm 2.80\end{array}$ & $\begin{array}{c}30^{\mathrm{a}} \\
\pm 4.35\end{array}$ & $\begin{array}{l}21^{\mathrm{a}} \\
\pm 4.35\end{array}$ & $\begin{array}{l}28.00^{\mathrm{a}} \\
\pm 5.29\end{array}$ & $\begin{array}{l}28.33^{a} \\
\pm 3.21\end{array}$ & $\begin{array}{l}27.66^{a} \\
\pm 8.00\end{array}$ & $\begin{array}{l}27.33^{\mathrm{a}} \\
\pm 2.51\end{array}$ & $\begin{array}{l}24.33^{a} \\
\pm 5.03\end{array}$ & $\begin{array}{c}23^{\mathrm{a}} \\
\pm 3.51\end{array}$ & 2.67 & ns \\
\hline
\end{tabular}

$\mathrm{a}, \mathrm{b}, \mathrm{c}$ Values within a row with different superscripts differ significantly; $*-p<0.05 ; * *-p<0.01$; ns-not significant.

1- T1 (L-carnitine NRC recommendations), T2 (L-carnitineNRC recommendations $+15 \%$ Lys and $15 \%$ Met), T3 (L-carnitineNRC recommendations $+15 \%$ Lys and $15 \% \mathrm{Met}), \mathrm{T} 4$ (75mg/Kg L-carnitine+Lys and MetNRC recommendations), T5 (75mg/Kg L-carnitine $+15 \%$ Lys and $15 \%$ Met), T6 (75mg/Kg L-carnitine $+30 \%$ Lys and $30 \% \mathrm{Met}), \mathrm{T} 7(150 \mathrm{mg} / \mathrm{Kg}$ L-carnitine + Lys and Met NRC recommendations), T8 (150mg/Kg L-carnitine + $15 \% \mathrm{Lys}$ and $15 \%$ Met), T9 (150mg/Kg L-carnitine $+30 \%$ Lys and $30 \%$ Met).

At day 42 , no significant changes were found in the mean blood parameters concentrations between birds submitted to extra Lys-Met supplementation without extra L-carnitine (T2 and T3) and the control group (T1). However, extra Lys-Met supplementation alone showed a discreet decay in plasmatic total cholesterol (TC) and low density lipoprotein (LDL) when compared with the control diet (T1) (Table 3). No main effects were evident for Lys plus Met supplementation across treatments (Table 4).

Table 4. Impact of the level of lysine (Lys) and methionine (Met) in the diet on blood parameters of broilers.

\begin{tabular}{|c|c|c|c|c|c|}
\hline \multirow{2}{*}{ Blood parameters } & \multicolumn{5}{|c|}{ Lys-Met } \\
\hline & $+0 \%$ & $+15 \%$ & $+30 \%$ & Sig & s.e.m. \\
\hline Glucose $[\mathrm{mg} / \mathrm{dl}]$ & $\begin{array}{l}245.66^{a} \\
\pm 19.71\end{array}$ & $\begin{array}{l}245.55^{a} \\
\pm 16.51\end{array}$ & $\begin{array}{c}238.00^{\mathrm{a}} \\
\pm 7.43\end{array}$ & ns & 5.23 \\
\hline Uric acid [mg/dl] & $\begin{array}{l}3.40^{\mathrm{a}} \\
\pm 1.83\end{array}$ & $\begin{array}{l}3.82^{\mathrm{a}} \\
\pm 1.38\end{array}$ & $\begin{array}{l}4.10^{\mathrm{a}} \\
\pm 1.44\end{array}$ & ns & 0.45 \\
\hline Triglyceride [mg/dl] & $\begin{array}{l}135.56^{\mathrm{a}} \\
\pm 15.92\end{array}$ & $\begin{array}{l}137.78^{\mathrm{a}} \\
\pm 21.78\end{array}$ & $\begin{array}{l}119.78^{\mathrm{a}} \\
\pm 28.23\end{array}$ & ns & 7.60 \\
\hline Protein [mg/dl] & $\begin{array}{l}3.28^{a} \\
\pm 0.49\end{array}$ & $\begin{array}{l}3.23^{a} \\
\pm 0.33\end{array}$ & $\begin{array}{l}3.44^{a} \\
\pm 0.42\end{array}$ & ns & 0.15 \\
\hline Albumin $[\mathrm{mg} / \mathrm{dl}]$ & $\begin{array}{l}1.43^{a} \\
\pm 0.19\end{array}$ & $\begin{array}{l}1.44^{\mathrm{a}} \\
\pm 0.21\end{array}$ & $\begin{array}{l}1.61^{\mathrm{a}} \\
\pm 0.20\end{array}$ & ns & 0.07 \\
\hline Cholesterol [mg/dl] & $\begin{array}{l}204.67^{a} \\
\pm 74.26\end{array}$ & $\begin{array}{l}177.44^{a} \\
\pm 86.26\end{array}$ & $\begin{array}{l}181.44^{\mathrm{a}} \\
\pm 68.59\end{array}$ & ns & 9.44 \\
\hline $\mathrm{LDL}[\mathrm{mg} / \mathrm{dl}]$ & $\begin{array}{l}87.22^{\mathrm{a}} \\
\pm 72.50\end{array}$ & $\begin{array}{l}65.22^{\mathrm{a}} \\
\pm 78.91\end{array}$ & $\begin{array}{r}66.22^{\mathrm{a}} \\
\pm 70.05\end{array}$ & ns & 9.24 \\
\hline $\mathrm{HDL}[\mathrm{mg} / \mathrm{dl}]$ & $\begin{array}{l}90.11^{\mathrm{a}} \\
\pm 8.57\end{array}$ & $\begin{array}{l}84.66^{a} \\
\pm 10.75\end{array}$ & $\begin{array}{l}91.33^{\mathrm{a}} \\
\pm 8.48\end{array}$ & ns & 3.14 \\
\hline $\operatorname{VLDL}[\mathrm{mg} / \mathrm{dl}]$ & $\begin{array}{l}27.33^{a} \\
\pm 3.31\end{array}$ & $\begin{array}{c}27.55^{a} \\
\pm 4.47\end{array}$ & $\begin{array}{l}23.88^{a} \\
\pm 5.71\end{array}$ & ns & 1.54 \\
\hline
\end{tabular}

a,bValues within a row with different superscripts differ significantly; ns-not significant. a través de tratamientos para ácido úrico plasmático (UAC), colesterol (TC), y lipoproteína de baja densidad (LDL), (Tabla 5).

Las menores concentraciones de UAc plasmático y las mayores concentraciones de TC y LDL plasmático se observaron en tratamientos en los que no se adicionó L-carnitine (T2 y T3), mientras que las mayores concentraciones de UAc plasmático y menores concentraciones de TC y LDL plasmático se observaron en los grupos suplementados con $150 \mathrm{mg} / \mathrm{kg}$ de L-carnitine (T7, T8 y T9).

Con relación a la concentración de ácido úrico plasmático (UAc), una diferencia significativa $(p<0.05)$ se encontró entre aves alimentadas con $150 \mathrm{mg} / \mathrm{kg}$ de L-carnitine más 30\% de Lys y Met (T9) y el grupo de control. Una diferencia estadística también se encontró para la concentración de lipoproteínas de alta densidad $(H D L, p<0.01)$ entre el grupo de control y las aves alimentadas con 150 $\mathrm{mg} / \mathrm{kg}$ de L-carnitine más $15 \%$ de Lys y Met (T8) (Tabla 3).

El colesterol total (TC) y la lipoproteína de baja densidad ( $L D L$ ) disminuyeron de manera significativa $(p<0.05)$ cuando se adicionó L-carnitine, con o sin Lys y Met. En grupos alimentados con $150 \mathrm{mg} /$ $\mathrm{kg}$ de L-carnitine más Lys y Met (T8 y T9), la concentración de colesterol plasmático (TC) fue significativamente menor $(p<0.05)$ que en grupos sin suplementación de L-carnitine (T1, T2, y T3) y en animales alimentados con $75 \mathrm{mg} / \mathrm{kg}$ de L-carnitine más $30 \%$ de Lys y Met (T4). Con relación a la concentración de LDL en grupos sometidos a 
The supplementation of the diet with L-carnitine and Lys plus Met had no effect $(p>0.05)$ in plasmatic glucose (GLU), triglyceride (TG), total protein (TP), albumin (Alb) and low density lipoprotein (VLDL). In contrary, the effect of treatments on plasmatic uric acid (UAC), cholesterol (TC), low density lipoprotein (LDL) and high-density lipoprotein (HDL) levels was significant $(p<0.05)$ (Table 3$)$. Main effects were evident for L-carnitine supplementation across treatments for plasmatic uric acid (UAC), cholesterol (TC) and low density lipoprotein (LDL), (Table 5).

The lowest concentrations in plasmatic UAC and the highest concentrations in plasmatic TC and LDL were observed in treatments where no L-carnitine was added (T2 and T3), whereas the highest concentrations in plasmatic UAc and the lowest concentrations in plasmatic TC and LDL were seen in the groups supplemented with 150 $\mathrm{mg} / \mathrm{kg}$ L-carnitine (T7, T8 and T9).

Concerning plasmatic uric acid (UAC) concentration, a significant difference $(p<0.05)$ was found between birds feed $150 \mathrm{mg} / \mathrm{kg}$ L-carnitine plus 30\% Lys and Met (T9) and the control group. A statistical difference was also found for high density lipoproteins $(H D L, p<0.01)$ concentration between the control group and the birds feed $150 \mathrm{mg} / \mathrm{kg}$ L-carnitine plus 15\% Lys and Met (T8) (Table 3).

The total cholesterol (TC) and low density lipoprotein ( $L D L)$ were significantly reduced $(p<0.05)$ when L-carnitine was added, with or without Lys and Met. In groups fed $150 \mathrm{mg} /$ $\mathrm{kg} \mathrm{L-carnitine} \mathrm{plus} \mathrm{Lys} \mathrm{and} \mathrm{Met} \mathrm{(T8} \mathrm{and} \mathrm{T9),}$ plasmatic cholesterol (TC) concentration was significant lower $(p<0.05)$ than in groups without L-carnitine supplementation (T1, T2 and T3) and in birds feed $75 \mathrm{mg} / \mathrm{kg} \mathrm{L}$-carnitine without Lys and Met (T4). As to, plasmatic LDL concentration in groups submitted to $75 \mathrm{mg} / \mathrm{kg}$ L-carnitine plus $30 \%$ Lys and Met (T6) and $150 \mathrm{mg} / \mathrm{kg}$ L-carnitine without and with Lys and Met (T7, T8 and T9), differed significantly $(p<0.01)$ from groups without L-carnitine supplementation ( $\mathrm{T} 1, \mathrm{~T} 2$ and T3) and in birds feed $75 \mathrm{mg} / \mathrm{kg}$ L-carnitine without and with 15\% Lys and Met (T4 and T5) (Table 3).

\section{DISCUSSION}

An exact characterization of the metabolites' profile in the birds of the various treatment groups is not possible since only one blood sample was collected at day 42 . However, using some markers of energy, protein and lipid metabolism such as glucose, cholesterol, triglyceride, uric acid, albumin and total
Table 5. Impact of the level of L-carnitine in the diet on blood parameters of broilers.

\begin{tabular}{|c|c|c|c|c|c|}
\hline \multirow[b]{2}{*}{ Blood parameters } & \multicolumn{5}{|c|}{ L-carnitine } \\
\hline & $\begin{array}{c}0 \\
(\mathrm{mg} / \mathrm{kg})\end{array}$ & $\begin{array}{c}75 \\
(\mathrm{mg} / \mathrm{kg})\end{array}$ & $\begin{array}{c}150 \\
(\mathrm{mg} / \mathrm{kg})\end{array}$ & Sig & s.e.m. \\
\hline Glucose[mg/dl] & $\begin{array}{c}236.00^{\mathrm{a}} \\
\pm 6.80\end{array}$ & $\begin{array}{l}246.11^{a} \\
\pm 15.70\end{array}$ & $\begin{array}{c}247.11^{a} \\
\pm 19.54\end{array}$ & ns & 5.23 \\
\hline Uric acid[mg/dl] & $\begin{array}{c}2.50^{a} \\
\pm 32.76\end{array}$ & $\begin{array}{l}4.06^{b} \\
\pm 1.32\end{array}$ & $\begin{array}{l}4.77^{\mathrm{b}} \\
\pm 1.41\end{array}$ & $* *$ & 0.45 \\
\hline Triglyceride[mg/dl] & $\begin{array}{l}129.56^{a} \\
\pm 25.05\end{array}$ & $\begin{array}{l}132.11^{\mathrm{a}} \\
\pm 28.40\end{array}$ & $\begin{array}{l}131.44^{a} \\
\pm 17.42\end{array}$ & ns & 7.60 \\
\hline Protein[mg/dl] & $\begin{array}{l}3.15^{\mathrm{a}} \\
\pm 0.26\end{array}$ & $\begin{array}{l}3.37^{a} \\
\pm 0.47\end{array}$ & $\begin{array}{l}3.42^{a} \\
\pm 0.47\end{array}$ & ns & 0.15 \\
\hline Albumin[mg/dl] & $\begin{array}{l}1.44^{\mathrm{a}} \\
\pm 0.21\end{array}$ & $\begin{array}{l}1.48^{\mathrm{a}} \\
\pm 0.20\end{array}$ & $\begin{array}{l}1.55^{\mathrm{a}} \\
\pm 0.22\end{array}$ & ns & 0.07 \\
\hline Cholesterol[mg/dl] & $\begin{array}{c}282.78^{a} \\
\pm 32.75\end{array}$ & $\begin{array}{c}153.33^{b} \\
\pm 35.59\end{array}$ & $\begin{array}{c}127.44^{b} \\
\pm 15.00\end{array}$ & $* *$ & 9.44 \\
\hline $\mathrm{LDL}[\mathrm{mg} / \mathrm{dl}]$ & $\begin{array}{l}164.22^{\mathrm{a}} \\
\pm 26.57\end{array}$ & $\begin{array}{l}38.00^{b} \\
\pm 40.17\end{array}$ & $\begin{array}{c}16.44^{b} \\
\pm 8.98\end{array}$ & $* *$ & 9.24 \\
\hline $\mathrm{HDL}[\mathrm{mg} / \mathrm{dl}]$ & $\begin{array}{l}92.66^{a} \\
\pm 10.01\end{array}$ & $\begin{array}{c}88.89^{a} \\
\pm 8.27\end{array}$ & $\begin{array}{c}84.55^{\mathrm{a}} \\
\pm 9.16\end{array}$ & ns & 3.14 \\
\hline VLDL[mg/dl] & $\begin{array}{c}25.88^{a} \\
\pm 5.20\end{array}$ & $\begin{array}{c}26.44^{a} \\
\pm 5.68\end{array}$ & $\begin{array}{c}26.44^{\mathrm{a}} \\
\pm 3.67\end{array}$ & ns & 1.54 \\
\hline
\end{tabular}
$a, b$ Values within a row with different superscripts differ significantly;
$* *-p<0.01$; ns-not significant.

$75 \mathrm{mg} / \mathrm{kg}$ de L-carnitine más $30 \%$ de Lys y Met (T6) y $150 \mathrm{mg} / \mathrm{kg}$ de L-carnitine con Lys y Met (T7, T8 y T9), difirieron significativamente $(p<0.01)$ de grupos con suplementación de L-carnitine ( $T 1$, T2 y T3) y en aves alimentadas con $75 \mathrm{mg} / \mathrm{kg}$ de L-carnitine sin y con $15 \%$ de Lys y Met (T4 y T5) (Tabla 3).

\section{DISCUSIÓN}

Una caracterización exacta del perfil de metabolitos en aves de los diversos grupos de tratamientos no se puede realizar debido a que sólo una muestra de sangre se recolecto en el día 42. Sin embargo, utilizando algunos marcadores de energía, proteína y metabolismo lípido como glucosa, colesterol, triglicéridos, ácido úrico, albúmina y proteína total, los cambios obvios en el metabolismo de carbohidratos, grasa y proteínas se pueden probablemente detectar. La interpretación de los resultados de la bioquímica de la sangre se deben combinar con el crecimiento total, peso de la carne, peso de la grasa y calidad de la carne para que sean útiles en el diseño de nuevas dietas. Sin embargo, los triglicéridos en suero (TG), colesterol total (TC), LDL y HDL son parámetro biométricos cruciales que reflejan el estatus del metabolismo lípido.

Es este estudio, la suplementación de Lys-Met per se, no tuvo efecto alguno en los parámetros de sangre en el día 42 pero en combinación con extra L-carnitine se observaron diferencias significativas entre los grupos para ácido úrico (UAc), colesterol total (TC), y concentraciones de LDL y HDL en plasma de pollos de engorde en el día 42. 
protein, the obvious changes in carbohydrate, fat and protein metabolism are likely detected. The interpretation of results of the blood biochemistry must be combined with total growth, meat weight, fat weight and meat quality in order to be useful for designing new diets. Nevertheless, serum triglyceride (TG), total cholesterol (TC), LDL, and HDL are crucial biochemical parameters reflecting the status of lipid metabolism.

In this study, the Lys-Met supplementation per si had no effect on blood parameters at day 42 but in combination with extra L-carnitine significant differences were observed among groups for the uric acid (UAC), total cholesterol (TC), LDL and HDL concentrations in broiler plasma at day 42 .

In broilers, uric acid (UAC) is produced as the main end product of nitrogen metabolism. The dietary protein level markedly affects uric acid production. Our results showed that UAc increase as dietary L-carnitine, Lys and Met intake increases. However, inconsistent results have been reported in the literature regarding the use of these amino acids in poultry diets. Donsbough et al (18) described a decrease in UAc when dietary Lys was increased in the diet while Xie et al (19) described a decrease in serum UAc concentrations followed by an increase as the dietary Met level increased.

In our study, concentration of protein and albumin were not affected by different levels of L-carnitine and Lys-Met. L-carnitine induced significant increases of serum albumin (Alb) concentrations in dietary energy depleted birds without affected total protein (20). Most studies on Met could not prove an effect on serum total protein and albumin in broilers $(13,21)$.

The plasmatic glucose (GLU) and free fatty acids concentrations are good indicators of the energetic status. The dynamic interactions between these two major energy substrate pools, without hormonal mediation seems to indicate that the utilization of one nutrient (e.g. glucose) directly inhibits the use of the other (in this case fatty acids) in accordance with Arslan et al (22).

Mitochondrial fatty acid $\beta$-oxidation is an important system by which fatty acids are broken down by various tissues to produce energy. In this system, L-carnitine plays an important role by facilitating the transport of fatty acids from cytosol across the inner mitochondrial membrane.

In our study, the concentration of glucose (GLU) and triglyceride (TG) was not affected by different levels of L-carnitine and Lys-Met in the diet. These results are in accordance with the study of Taraz and Dastar (14) since they showed that the concentration of
En pollos de engorde, el ácido úrico (UAC) se produce como el producto final principal del metabolismo del nitrógeno. El nivel de proteína dietaria afecta marcadamente la producción de ácido úrico. Nuestros resultados mostraron que el UAc incrementó a medida que la ingesta de L-carnitine, Lys y Met disminuyó. Sin embargo, se han reportado resultados inconsistentes en la literatura con relación al uso de estos aminoácidos en las dietas de aves. Donsbough et al (18) describió una disminución en el UAc cuando se incrementó el Lys dietario en la dieta mientras que Xie et al (19) describió una disminución de las concentraciones de UAc en suero seguido de un incremento a medida que se incrementaba el nivel de Met.

En nuestro estudio, la concentración de proteína y albúmina no fue afectada por los diferentes niveles de L-carnitine y Lys-Met. L-carnitine indujo incrementos significativos de concentraciones de albúmina (Alb) en suero en aves con depleción de energía dietaria sin afectar la proteína total (20). La mayoría de los estudios sobre Met no pudieron probar un efecto de proteína total en suero y albúminas en pollos de engorde $(13,21)$.

La glucosa plasmática (GLU) y concentraciones de ácidos grasos libres son buenos indicadores del estatus energético. Las interacciones dinámicas entre estos dos grupos de sustratos de energía principales, sin mediación hormonal, parece indicar que la utilización de un nutriente (ej. Glucosa) directamente inhibe el uso del otro (en este caso ácidos grasos) de acuerdo con Arslan et al (22).

La $\beta$-oxidación de ácidos grasos mitocondriales es un sistema importante mediante el cual se descomponen los ácidos grasos en distintos tejidos para producir energía. En este sistema, L-carnitine juega un rol importante facilitando el transporte de ácidos grasos de citosol a través de la membrana mitocondrial.

En nuestro estudio, la concentración de glucosa (GLU) y triglicéridos (TG) no fue afectada por los distintos niveles de L-carnitine y Lys-Met en la dieta. Estos resultados están de acuerdo con el estudio de Taraz y Dastar (14) ya que mostraron que la concentración de GLU y TG no era afectada por suplementación de L-carnitine y Lys-Met. Así mismo, Arslan et al (22) no pudo encontrar un efecto de L-carnitine sobre las concentraciones de GLU plasmático. Se reportaron resultados en conflicto de Bouyeh y Gevorgyan (6). Sin embargo, nuestros resultados están en contra de Xu et al (4). Estos autores describen que L-carnitine causa una disminución en la concentración plasmática de triglicéridos 
GLU and TG was not affected by L-carnitine and Lys-Met supplementation. As well, Arslan et al (22) could neither find an effect of L-carnitine on plasmatic GLU concentrations. Conflicting results were reported by Bouyeh and Gevorgyan (6). However, our results are against the result of $\mathrm{Xu}$ et al (4). These authors described that L-carnitine causes the decrease of the plasmatic concentration of triglyceride (TG). Moreover, they found that by adding 50,75 , or $100 \mathrm{mg} / \mathrm{kgL}$-carnitine to diets the total activities of NADPH-generating enzymes (G-6-PD, $M D H$, and ICD) in subcutaneous fat were decreased $(p<0.05)$ and the synthesis of fatty acid in subcutaneous fat would accordingly be decreased.

In the present study, the concentration of $\mathrm{TC}$, LDL and HDL were affected by different levels of L-carnitine and Lys-Met, whereas VLDL plasma concentrations were not affected. Bouyeh and Gevorgyan (6) observed a decrease followed by an increase in plasma cholesterol in birds feed Lys and Met above NRC recommendations. It is possible that this was caused by increased cholesterol storage in muscles. Cholesterol is probably less actively stored and need a longer period of ingestion before significant increase or decrease may show up (6). In this study, the plasmatic total cholesterol (TC) and LDL were significantly reduced when L-carnitine was added, with or without Lys and Met. These results agree with those from Murali et al (23). However, the data by Taraz and Dastar (14), Adabi et al (24) and Arslan et al (22) do not agree with our findings.

The very low density lipoprotein (VLDL) and LDL are closely related to one another. VLDL is formed in the liver and is converted to LDL in muscle and adipose tissues by losing fatty acids. The LDL thus produced supply the tissues with cholesterol. The TG accumulation in adipocytes depends mainly on the availability of plasma VLDL and genetically fat chickens presented high levels of VLDL. In this study, despite no differences were found between treatments, the plasmatic VLDL concentration were low.

Taken together, our data show that a diet containing $150 \mathrm{mg} / \mathrm{kg}$ L-carnitine with 15\% Lys and 15\% Met seems to be enough to sustain low levels of TC, LDL and HDL. Therefore, L-carnitine may decrease body fat content in broiler carcasses by decreasing the deposition of fatty acids in extra hepatic tissues, above all the adipose and muscle tissue.

\section{Acknowledgments}

Financial support by Rasht Branch, Islamic Azad University, grant number 4.5830 is gratefully acknowledged.
(TG). Más aún, encontraron que al adicionar $50,75,0100 \mathrm{mg} / \mathrm{kg}$ de L-carnitine a las dietas las actividades de enzimas generadoras de NADPH (G-\&-PF, MDH e ICD) en grasa subcutánea disminuían $(p<0.05)$ y la síntesis de ácidos grasos en grasa subcutánea disminuía acordemente.

En este estudio, la concentración de TC, LDL y HDL fue afectada por los distintos niveles de L-carnitine y Lys-Met, mientras que las concentraciones de VLDL en plasma no fueron afectadas. Bouyeh y Gevorgyan (6) observaron una disminución seguida de un aumento en el colesterol en plasma en aves alimentadas con Lys y Met por encima de las recomendaciones de NRC. Es posible que esto haya sido causado por un incremento del almacenamiento de colesterol en los músculos. El colesterol es probablemente almacenado de manera menos activa y necesita un mayor periodo de ingestión antes que se evidencie una disminución o aumento significativo (6). En este estudio, el colesterol total plasmático (TC) y LDL disminuyeron significativamente cuando se adicionó L-carnitine, con o sin Lys y Met. Estos resultados están de acuerdo con aquellos de Murali et al (23). Sin embargo, los datos de Taraz y Dastar (14), Adabi et al (24) y Arslan et al (22) no están de acuerdo con nuestros hallazgos.

La lipoproteína de muy baja densidad (VLDL) y el LDL están relacionados de manera cercana entre sí. El VLDL se forma en el hígado y se convierte a LDL en el músculo y tejidos adiposos al perder ácidos grasos. El LDL producido proporciona a los tejidos con colesterol. La acumulación de TG en adipocitos depende principalmente de la disponibilidad de VLDL plasmático y pollos genéticamente gordos presentan altos niveles de VLDL. En este estudio, a pesar de que no se encontraron diferencias entre los tratamientos, las concentraciones de VLDL plasmático fueron bajas.

Tomados en conjunto, nuestros datos muestran que una dieta que contiene $150 \mathrm{mg} / \mathrm{kg}$ de L-carnitine con $15 \%$ de Lys y Met parece ser suficiente para mantener bajos niveles de TC, LDL y HDL. Por ende, L-carnitine puede reducir el contenido de grasa del cuerpo en carcasas de pollos de engorde disminuyendo la deposición de ácidos grasos en tejidos hepáticos extra, por encima de todo el tejido adiposo y muscular.

\section{Agradecimientos}

Por el apoyo financiero por Rasht Branch, Universidad Islámica Azad, número de concesión 45830. 


\section{REFERENCES}

1. Hamerschlag K. Meat Eaters Guide to Climate Change + Health. Environmental Working Group. Meat Eaters Guide: Report 2011. [cited 2014 Mai 22] Avaible from:http:// static.ewg.org/reports/2011/meateaters/ pdf/report_ewg_meat_eaters_guide_to_ health_and_climate_2011.pdf

2. Petracci M, Mudalal S, Bonfiglio A, Cavani C.Occurrence of white striping under commercial conditions and its impact on breast meat quality in broiler chickens. Poult Sci 2013; 92(6):1670-1675.

3. Cave MC, Hurt RT, Frazier TH, Matheson PJ, Garrison RN, McClain CJ et al. Obesity, inflammation, and the potential application of pharmaconutrition. Nutr Clin Pract 2008;23:16-34.

4. Xu ZR, Wang MQ. Effects of L-carnitine on growth performance, carcass composition, and metabolism of lipids in male broiler. Poult Sci 2003;82:408-413.

5. Kidd MT, Gilbert J, Corzo A, Page C, Virden WS, Woodworth JC. Dietary L-carnitine influences broiler thigh yield. Asian-Aust J Anim Sci 2009; 22(5):681-685.

6. Bouyeh M, Gevorgyan O. Influence of excess lysine and methionine on cholesterol fat and performance of broiler chicks. J Anim Vet Adv 2011; 10(12):1546-1550.

7. Celik L, Ozturkcan O, InalL TC, Canacankatan $\mathrm{N}$, Kayrin L. Effects of L-carnitine and niacin supplied by drinking water on fattening performance, carcass quality and plasma L-carnitine concentration of broiler chicks. Arch Anim Nutr 2003;57(2):127-136.

8. Mukhtar MA, Mohammed KA, Musa $\mathrm{MH}$.Replacement value of lysine and methionine for super concentrate in broiler chick's yield and quality. J Sc Tech 2010; 11(2):27-29.

9. Si J, Kersey JH, Fritts CA, Waldroup PW. An evaluation of the interaction of lysine and methionine in diets for growing broilers. Int J Poult Sci 2004; 3(1):51-60.
10. Corduk M, Ceylan N, Ildiz F. Effects of dietary energy density and L-carnitine supplementation on growth performance, carcass traits and blood parameters of broiler chickens. South African J Anim Sci 2007; 37(2):65-73.

11. Parizadian B, Ahangari YJ, Shargh MS, Sardarzadeh A. Effects of Different levels of L-carnitine supplementation on egg quality and blood parameters of laying japanese quail. Intern J Poult Sci 2011; 10(8):621-625.

12. Arslan C. L-carnitine and its use as a feed additive in poultry feeding a review. Revue Méd Vét2006; 157(3):134-142.

13. Al-Mayah AAS. Immune response of broiler chicks to DL-methionine supplementation at different ages. Int J Poult Sci 2006; 5:169-172.

14. Taraz Z, Dastar A. Effect of L-carnitine supplementing diets with different levels of protein on performance and blood parameters in broiler chickens. J Agric Sci Nat Res2008; 15(5):114-122.

15. Etim NAN, Akpabio U, Okpongete RO, Offiong EEA. Do Diets Affect Haematological Parameters of Poultry? British J Appl Sci \& Techn 2014; 4(13):1952-1965.

16. National Research Council (NRC). Nutrient Requirements of Poultry: Ninth Revised Edition. Washington, DC: The National Academies Press, 1994.

17. SAS, 2000. Statistical Analysis System. SAS User Guide: Release 9.2. SAS Institute INC, Cary NC, USA, 2000.

18. Donsbough AL, Powell $S$, Waguespack $A$, Bidner TD, Southern LL. Uric acid, urea, and ammonia concentrations in serum and uric acid concentration in excreta as indicators of amino acid utilization in diets for broilers. Poult Sci 2010; 89:287-94.

19. Xie M, Hou SS, Huang $W$, Zhao L, Yu JY, $\mathrm{Li} W Y$ et al. Interrelationship between methionine and cysteine of early peking ducklings. Poult Sci 2004; 83:1703-1708. 
20. Çakir S, Yalçin S Effects of L-carnitine supplementation in diets with low or normal energy level on growth performance and carcass traits in broilers. Revue Méd Vét 2007; 158(6):291-296.

21. Chattopadhyay K, Mondal MK, Roy B. Comparative efficacy of DL-methionine and herbal methionine on performance of broiler chicken. Int J Poult Sci 2006; 5:1034-1039.

22. Arslan C, Citil M, Saatci M. Effects of L-carnitine administration on growth performance, carcass traits, serum lipids and abdominal fatty acid compositions of geese. Rev Med Vet 2004: 155(6):315-320.
23. Murali $P$, George SK, Mercy AD, Dipu MT, Narayanankutty K. Effects of L-carnitine supplementation on serum lipid profile of broiler chicken fed with animal fat. Int J Agric and Food Sci Technol 2013; 4(8):773-774.

24. Adabi ShG, Moghaddam Gh, Taghizadeh A, Nematollahi A, Farahvash T. Effect of L-carnitine and vegetable fat on broiler breeder fertility, hatchability, egg yolk and serum cholesterol and triglyceride. Int Poult Sci 2006; 5(10):970-974. 